\title{
The Combination of Techniques for Automatic Semantic Image Annotation Generation in the IMAGINATION Application ${ }^{\star}$
}

\author{
Andreas Walter ${ }^{1}$ and Gabor Nagypal ${ }^{2}$ \\ ${ }^{1}$ FZI Research Center for Information Technologies , Information Process Engineering, \\ Haid-und-Neu-Straße 10-14, 76131 Karlsruhe, Germany \\ Andreas. Walterafzi.de \\ 2 disy Informationssysteme GmbH, Erbprinzenstr. 4-12, Eingang B, 76133 Karlsruhe, Germany \\ nagypal@isy.net
}

\begin{abstract}
The IMAGINATION project provides image-based navigation for digital cultural and scientific resources. Users can click on parts of an image to find other, interesting images to a given context. In this paper, we present the core parts of the IMAGINATION application. To allow the navigation through images, this application automatically generates high quality semantic metadata. Therefore it combines automated processes for person and object detection, face detection and identification in images together with text mining techniques that exploit domain specific ontologies.
\end{abstract}

\section{Introduction}

State of the art systems for the navigation through images either assume that users manually create semantic image annotations or they use automated processes for image annotation creation in isolation. SemSpace [1] uses domain ontologies and allows the semantic annotation of image parts. This application does not use automated processes at all. Thus, the annotation of images is a time consuming task in this system. Riya [2] uses a face detection algorithm. This reduces the annotation time for users, but leads to new problems. The sole usage of face detection algorithm leads to the problem of incorrectly generated annotations. E.g., so-called "phantom faces" may be generated.

The goal of IMAGINATION is to minimize the human effort to create high-quality image annotations. First, the annotation time for the creation of semantic images has to be reduced by automatic generation. Second, these generated artifacts must be of highquality to reduce the time needed for manual corrections. To achieve these goals, IMAGINATION combines different automated processes. Moreover, background knowledge in a common domain ontology is also exploited to achieve best result.

In Section 2, we present the automated processes that are used in the IMAGINATION application. In Section 3, we present a sample scenario to demonstrate the interaction of the processes and how this interaction helps increase to quality of annotations.

\footnotetext{
* This work was co-funded by the European Commission within the project IMAGINATION.
} 


\section{Components of the IMAGINATION Application}

In this section, we present the components of the IMAGINATION application. They work together to automatically generate semantic image annotations of the highest possible quality.

\subsection{ImageNotion - Collaborative Generation of Domain Ontologies}

Domain ontologies used in IMAGINATION base on the ImageNotion concept [3]. Ontologies according to ImageNotion consist of imagenotions. An imagenotion (formed from the words image and notion) graphically represents a semantic notion through an image. Furthermore, similarly to many existing ontology formalisms, it is possible to associate descriptive information, e.g. textual labels and date information with an imagenotion. Further, it is possible to add links to related web pages for an imagenotion. Links can help text mining algorithms to gather background information from web pages. In addition, relations between imagenotions are also supported. To achieve maximal understandability, ImageNotion makes no distinction between concepts and instances. Based on the ImageNotion methodology, users of the IMAGINATION system can collaboratively generate required domain ontologies. Also, it is possible to import and extend existing ontologies, such as CIDOC-CRM ([4]). Semantic annotations can be created either for the whole image or for image parts. Fig. 1 shows an the imagenotion for Manuel Barroso, the current president of the EU commission.

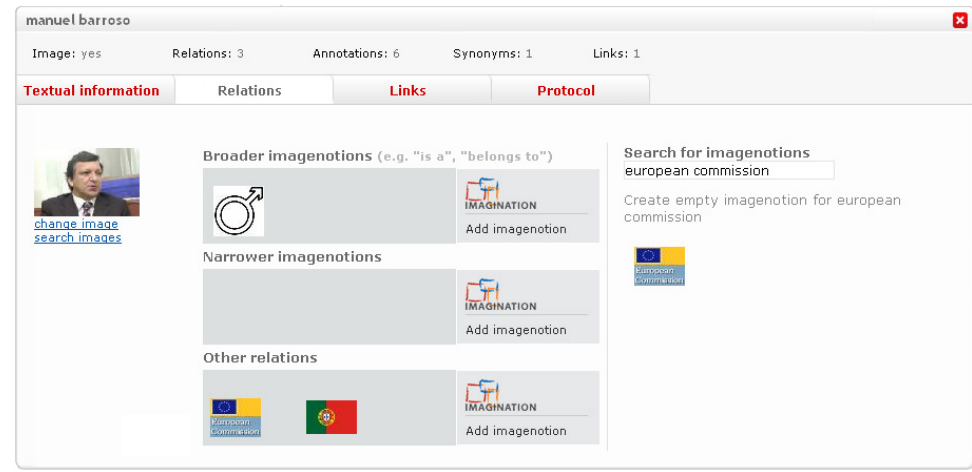

Fig. 1. Imagenotion for Manuel Barroso

\subsection{Automated Processes}

Most images contain text base image annotations. The text mining algorithms of JS 1 [5] allow the detection of semantic elements in such text based image annotations. They can also new ontology elements. Especially for this task, the background texts that are stored at imagenotions as links to external web pages are very useful.

\footnotetext{
${ }^{1}$ Links to partner web pages can be found on the project web site: http://www.imagination-project.org
} 
Face detection and identification algorithms are provided by Fraunhofer IIS ([6]). The face detection algorithm can detect parts on images that display faces. In addition, gender-classification of a detected person is also possible. The face identification algorithm aims to detect the relevant person for a detected face. It returns a list of proposals for the person and their corresponding relevance.

Person and object detection algorithms are provided by NTUA. Person detection finds the image area showing a person or a part of it. Object detection algorithms can identify objects, e.g. tanks, airplanes or cars.

\subsection{Controller for the Generation of Automatically Generated Image Annotations}

The controller for the generation of automatically generated image annotations gets an image as input. First, it loads available textual and manually created semantic annotations for the image. Then, it iteratively invokes the available automated processes one by one. Each of the automated processes can read and change the available annotations and image regions, and add new ones. This allows the correction or the refinement of existing result. E.g., face detection algorithms may thoroughly examine areas for faces that were detected as persons by the person detection algorithms. In addition, the text mining results may help to eliminate wrong suggestions in the face identification step. The controller stops the annotation process, when there are no further changes in an iteration.

\section{An Example Scenario for Using the IMAGINATION Application}

In this section we present an example scenario for using the IMAGINATION application. The input is an image showing the current president of the EU commission, Manuel Barroso, together with the former president of the EU commission, Romano Prodi.

First, the user uploads the image. Then, it is possible to create manual annotations. In Fig. 2 a user has added the imagenotion "Manuel Barrosso" for the complete image with a high rating.

In the next step, the user can start the automated annotation process. Fig. 3 shows the result of the automated processes. In the first iteration, the text mining algorithm has created the semantic annotations "Romano Prodi" and "Manuel Barroso", based on the textual title of the image: "EU president Barroso meets Prodi". The person and object detection algorithms have created two image annotations for the shapes of the two persons. The face detection algorithm has created two image annotations for the detected faces. The face identification has identified "Manuel Barroso" with a score of 80 percent and "Guenther Verheugen" with a score of 20 percent. Also, the gender of the second face was detected as "male". The controller now initiates another annotation round. In this round, the person identification can use the results of the other automated processes. For the second face, it creates a new image annotation "Romano Prodi" for Prodi's face with a score of 100 percent and sets the score of "Guenther Verheugen" to zero, since the information of the text mining algorithm and the manual annotation 


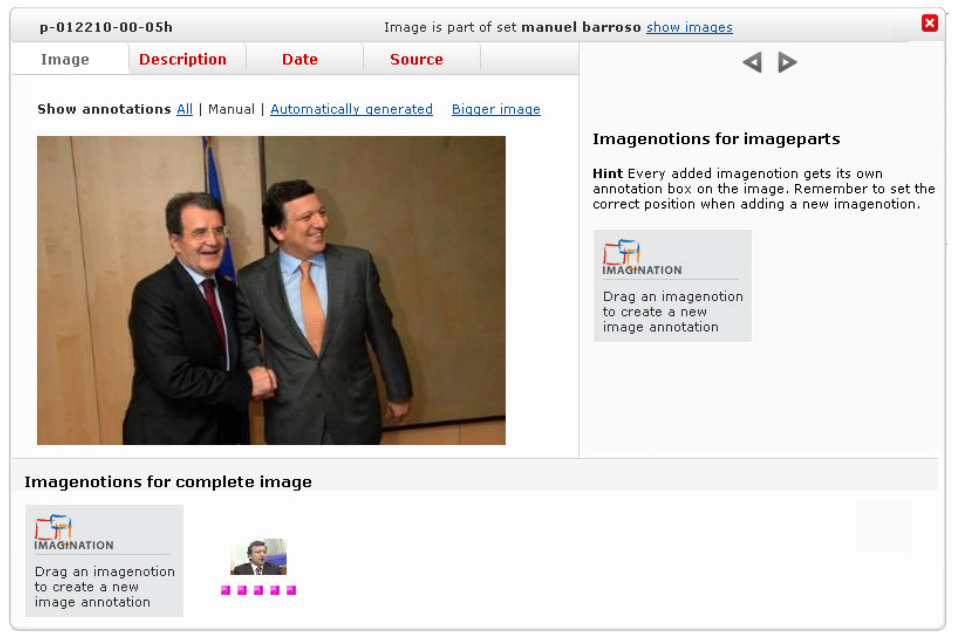

Fig. 2. Manual annotation of an image

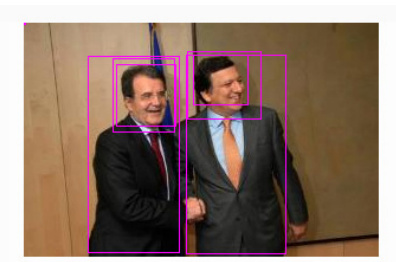

Show protocoll

Controll processes

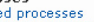

Show seperated results of processes

Face detection and identification

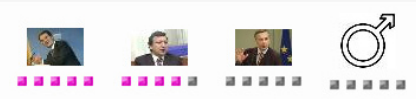

Object and person detection

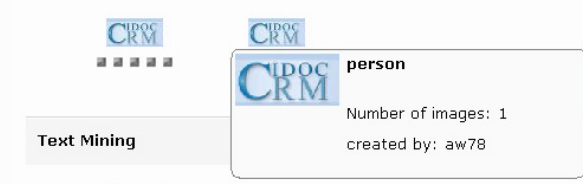

Fig. 3. Results of the automated processes

states, that there must be the person "Manuel Barroso". Also, it sets the score of the detected areas for the persons to zero, since two faces were detected. In the third iteration round, there are no more changes and the controller returns the result.

${ }^{2}$ Our users prefer faces to body contours, when the size of the face is big enough. 


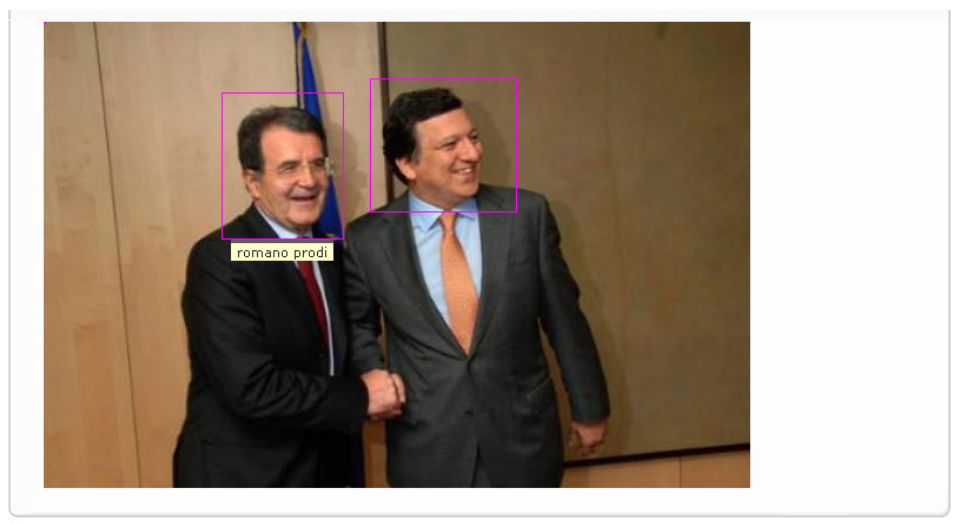

Fig. 4. Resulting semantic image annotations for the image

Fig. 2] shows the final result of the annotation when a user searches for the image. The result contains the detected annotation boxes of "Manuel Barroso" and "Romano Prodi". Thus, the combination of the automated processes with domain ontologies lead to automatically created image annotations with a higher quality than using the automated processes in isolation.

The application is accessible at www.imagenotion.com

\section{References}

1. van Ossenbruggen, J., Troncy, R., Stamou, G., Pan, J.Z.: Image Annotation on the Semantic Web. W3C working draft, W3C (2006)

2. Riya: Riya - Visual search (2007) (accessed 2007-12-09), http: / /www.riya.com/

3. Walter, A., Nagypal, G.: Imagenotion - methodology, tool support and evaluation. In: GADA/DOA/CoopIS/ODBASE 2007 Confederated International Conferences DOA, CoopIS and ODBASE, Proceedings. LNCS, Springer, Heidelberg (2007)

4. Crofts, N., Doerr, M., Gill, T., Stead, S., Stiff, M.: Definition of the cidoc conceptual reference model version 4.2. In: CIDOC CRM Special Interest Group (2005)

5. Fortuna, B., Grobelnik, M., Mladenic, D.: Semi-automatic data-driven ontology construction system. In: Proc. of the 9th International multi-conference Information Society IS-2006, Ljubljana (2006)

6. Küblbeck, C., Ernst, A.: Face detection and tracking in video sequences using the modified census transformation. In: Baker, K.D. (ed.) Image and Vision Computing, vol. (6), Elsevier, Amsterdam (2006) 